\title{
El ENFOQUE CUALITATIVO EN EL ESTUDIO DE REPRESENTACIONES SOBRE EL TRABAJO EN SUJETOS PRIVADOS DE LA LIBERTAD ${ }^{1}$ A QUALITATIVE APPROACH TO STUDY REPRESENTATIONS ABOUT WORK IN SUBJECTS DEPRIVED OF FREEDOM
}

\section{Resumen}

La presente investigación busca explorar y describir los cambios operados en las representaciones psíquicas del trabajo de un grupo de internos ${ }^{4}$, insertos en un dispositivo laboral institucional. Es un estudio cualitativo que utiliza como método la historia de vida y la técnica administrada es la entrevista semidirigida. Brinda, además, un aporte a la administración desde un enfoque cualitativo y la aplicación poco usual, en este ámbito de conocimiento, del método historia de vida. Se examinan cinco internos por medio de entrevistas en profundidad en diferentes momentos cronológicos de su inserción en el programa laboral. Los resultados obtenidos brindan elementos a los directivos, o gestores, para la definición y desarrollo de políticas de tratamiento penitenciario,

1 Este artículo constituye un resumen de la tesis de doctorado en Ciencias de la Administración (UNS) de Fernando Menichelli, dirigida por el Dr. Gastón Milanesi. La misma fue defendida y aprobada el 12 de marzo de 2019.

2 Universidad Nacional del Sur, Departamento de Ciencias de la Administración (UNS-DCA). fmenichelli@uns.edu.ar

3 Universidad Nacional del Sur, Departamento de Ciencias de la Administración (UNS-DCA). milanesi@uns.edu.ar

4 Interno es aquel sujeto privado de su libertad por encontrarse condenado o procesado por una causa penal. 
como así también elementos valiosos para el desarrollo de prácticas ajustadas en recursos humanos a las diferentes empresas que trabajan con estos grupos.

Palabras clave: cambios, representaciones, trabajo, internos, cárcel.

\begin{abstract}
This research seeks to explore and describe changes in the psychic representations about work of a group of inmates ${ }^{5}$ participating in an institutional work program. Based on a qualitative approach, this study uses life history as a research method and semi-directed interview as a data collection technique. Applying life history in administration is meant to be a contribution to the field. Five inmates are examined through in-depth interviews at different chronological times during their participation in the work program. The results obtained provide both executives, or managers, and companies involved with such groups with relevant information either to define and develop prison treatment policies or to develop human resource practices.
\end{abstract}

Keywords: changes, representations, work, inmates, prison.

JEL: J68, M14, M51.

5 Internal is a person deprived of his liberty for being convicted or prosecuted in a criminal case. 
El enfoque cualitativo en el estudio de representaciones sobre el trabajo en sujetos...

\section{Introducción}

\subsection{Relevancia social y pertinencia científica del tema}

La elección del presente tema de investigación se debe a la inquietud de vincular los temas de gestión con un ámbito donde, si bien la gestión ocurre, no ha sido estudiada ni aplicada en todo su potencial como lo son los sistemas carcelarios. En este contexto es que el interés surge en poder explorar y caracterizar las representaciones psicológicas del trabajo de un grupo de internos y brindar la posibilidad de que ellos se expresen sobre las prácticas de tratamiento penitenciario de las cuales son objeto.

En una vertiente del análisis sobre la relevancia del tema, se encuentra el incremento del accionar delictivo o de lo que ha sido pasible de sanción penal en nuestra sociedad actual, en particular en la ciudad de Bahía Blanca. Se explica el aumento del accionar delictivo concibiéndolo como producto y productor de la anomia social. De este modo es legitimado y perpetuado por las instituciones que lo tratan y la sociedad en su conjunto, legitimando lo criminalizable. En concordancia con ello, Volnovich (2000) caracteriza a los excluidos del sistema como depositarios del malestar de la sociedad.

La relevancia del tema se funda por un lado en su originalidad, dado que no hay información sobre los efectos de los programas laborales de reinserción en el Servicio Penitenciario Bonaerense, y por otro lado en que brinda información desde la mirada de aquellos sobre quienes se realizan las prácticas de tratamiento penitenciario actuales permitiendo la ratificación y/o mejora de las mismas.

El trabajo propuesto, en consecuencia, busca evitar la toma de posiciones reduccionistas, dando cuenta de las múltiples causas de la problemática de la reinserción social de internos que se expresa en nuestra sociedad actual, así como contribuir al avance en el campo de la investigación en psicología y tratamiento penitenciario brindando conocimientos a los administradores.

\subsection{El objetivo del trabajo}

A través de la realización de este trabajo se busca estudiar el enfoque metodológico pertinente y necesario para comprender las representaciones psicológicas de un grupo de internos que participan de un programa laboral extramuros. 
El objetivo es brindar, de esta manera, elementos para la gestión tanto a los dirigentes de estas organizaciones como así también a cada integrante del dispositivo, partiendo de la perspectiva de los destinatarios. Además se intentará aportar propuestas de trabajo con internos en el ámbito del Servicio Penitenciario Bonaerense.

\section{Marco teórico}

\subsection{El tratamiento en el Servicio Penitenciario y los dispositivos laborales}

A continuación se describirá a qué se denomina tratamiento penitenciario y cuáles son sus características, y la especie particular que se describe en este trabajo. El tratamiento penitenciario es conceptualizado como un conjunto de prácticas institucionalizadas y previstas en las leyes nacionales, como así también en las leyes y reglamentaciones del Servicio Penitenciario Bonaerense.

La Constitución Nacional Argentina (sancionada en 1853 y con reformas en los años 1860, 1866, 1898, 1957 y 1994) subraya, en el artículo 18, el carácter resocializador de las cárceles, sosteniendo que no son dispositivos creados para el castigo, sino un instrumento de seguridad y readaptación social, y responsabilizando a los jueces a cargo de los individuos privados de la libertad si ello no se cumpliera.

La ley orgánica del Servicio Penitenciario Bonaerense (Decreto Ley 9079 y sus modificatorias) establece en su artículo primero que se trata de una fuerza de seguridad que contribuye al mantenimiento del orden público y colabora en la obtención de la paz social, siendo su misión la custodia y la guarda de los procesados y la ejecución de las sanciones penales privativas de la libertad, como así también todo acto que se imponga por leyes, decretos y resoluciones (artículo segundo). A su vez, en el artículo tercero, inciso b de dicho texto legal, se introduce mediante modificación la función de posibilitar la readaptación social de los condenados.

Por otra parte, en el artículo cuarto de la Ley de Ejecución Penal Bonaerense 12256, se establece como fin de dicha normativa la adecuada inserción social de los procesados y condenados a través de la asistencia o tratamiento y control. El artículo séptimo del mismo texto prevé la asistencia de procesados y la asistencia y o tratamiento de condenados, que se brindará mediante la implementación de programas específicos destinados a hacer efectivos los 
El enfoque cualitativo en el estudio de representaciones sobre el trabajo en sujetos...

derechos y garantías constitucionales. El artículo octavo menciona que se tenderá a reducir las diferencias entre la vida en prisión y la vida por fuera de la institución, buscando de forma activa la preservación de los vínculos con las diferentes dimensiones de la vida en libertad - familiar, educacional y laboral-, pudiendo buscar la cooperación de instituciones de la comunidad, organismos estatales, privados, nacionales o extranjeros.

El dispositivo laboral en el que se enfoca nuestro estudio es inédito en el Servicio Penitenciario Bonaerense (Programa de Reinserción Laboral); su comienzo data del 12 de octubre de 2007. Este programa ha sido viable a partir de acuerdos de cooperación, y ha sido declarado de interés por instituciones y organismos tales como la Municipalidad de Bahía Blanca y el Honorable Concejo Deliberante, los Ministerios de Trabajo y Educación de la Provincia de Buenos Aires y la Confederación General del Trabajo y la Unión Industrial.

El Programa de Reinserción Laboral se encuentra previsto en lo que se denomina tratamiento penitenciario. Se diferencia de las actividades históricas de tratamiento penitenciario en tanto consiste en la realización de una actividad de tipo laboral en un contexto amplio, por fuera de la unidad carcelaria. Es así como los internos que participan en este programa laboral de resocialización se incluyen en empresas de diferentes rubros del contexto social local.

\subsection{Breve reseña histórica}

$\mathrm{Al}$ caracterizar el sistema carcelario es necesario destacar distintas concepciones que históricamente se le han atribuido. Estas concepciones han ido definiendo diferentes modos de acercarse a este objeto para investigarlo. La problemática inicial que se formula con relación al objeto, por lo tanto, es amplia, y se volverá progresivamente más focalizada durante el proceso de investigación. Como señala Sirven (1999), el trabajo en el campo permite ajustar la lente a partir de preguntas emergentes específicas.

Se busca, desde una revisión crítica de las prácticas y de sus significados, dar cuenta de los procesos socio histórico y político que dieron origen al objeto de conocimiento: la cárcel. En la actual investigación, estos procesos constituyen el contexto del objeto de estudio. Para abordarlo científicamente, se pondrán de relieve los aspectos pertinentes de las disciplinas que han teorizado sobre ella en diferentes momentos; tal posicionamiento supone comprender la cárcel y sus prácticas por medio de la contextualización histórica, en la búsqueda de desnaturalizar lo existente, lo que se vive como ineludible, como 
dado y evidente. Es necesario, en esta misma línea de pensamiento, concebir el proceso de institucionalización de la cárcel como algo construido artificialmente por mentes y prácticas humanas, lo cual permite el cuestionamiento, reconstrucción y transformación por otras mentes, otras intencionalidades y otras prácticas humanas.

Para comprender en el presente un fenómeno social es fundamental ligar lo actual con su etiología, siendo importante hacer historia en la temática de la exclusión y sus efectos. Ya Marx (2011 [1848]) plantea, al describir el surgimiento de la clase trabajadora, que en el siglo El se constituye una parcela significativa de trabajadores excluidos del proceso productivo, a los cuales se los define como ejército de reserva de la fuerza de trabajo; ese residuo de superpoblación que vegeta en la pauperización, o parcela degradada del proletariado, son los llamados criminales, vagabundos y prostitutas.

En este punto, se puede reflexionar acerca de los significados que adquieren los conceptos 'vigilar' y 'castigar' para Foucault (1989); así se abre el interrogante: ¿cómo caracteriza Foucault los modos organizativos? El autor hace foco en que el castigo en el régimen político de la monarquía - cualquiera fuese el atentado contra lo que estaba establecido - asumía, por excelencia, la forma del suplicio. Foucault también aborda la cuestión desde los orígenes de los "derechos humanos", los cuales tienen su nacimiento en la Declaración de los Derechos Universales del Hombre, sentenciada en la Primera República Francesa. Esa nueva ideología fue el fundamento para este nuevo posicionamiento o planteo, contrario al de las prácticas monárquicas existentes en ese momento histórico.

\subsection{Dimensión interinstitucional e interinstitucional}

Otros aspectos que adquieren relevancia al momento de pensar la problemática de la cárcel, y qué hacer con estos sujetos privados de su libertad, son los factores interinstitucionales y los factores interinstitucionales.

A nivel interinstitucional se puede observar una realidad particular producida por el aislamiento, la falta de recursos humanos, las dificultades relativas a la incorporación de personal, su capacitación y desarrollo. Así también, adquiere relevancia la contaminación interinstitucional del personal por las pautas tácitas e implícitas del sistema carcelario: actitudes, formas de hablar, conductas, lo cual define una cultura organizacional que puede ser pensada como un mecanismo de adaptación del personal a la institución. También es 
El enfoque cualitativo en el estudio de representaciones sobre el trabajo en sujetos...

destacable la variable económica, el gran presupuesto asignado a la creación de cárceles para evitar "la destrucción social". Esta erogación de recursos no suele destinarse a inversión en resocialización, sino que muchas veces significa un costo social a pagar por las fallas inherentes al sistema; tal condición tiende a mantener el statu quo social e institucional.

Con relación a la dimensión tecnológica: falta de recursos y equipamiento actualizados para el personal y un adecuado y actualizado soporte administrativo que facilite y mejore la dinámica y eficiencia institucional. Estos factores complejizan aún más la situación a la hora de analizar la "delincuencia", su reincidencia y la posibilidad de reinserción social, abriéndose la pregunta de si se trata de reinsertar o insertar, y a dónde.

"La población carcelaria ha cambiado", expresan los internos de antigua data. Con esta afirmación resaltan que hubo otras épocas en las cuales los delincuentes tenían códigos, u otros códigos: "no se le robaba al trabajador, ahora se drogan y hacen estragos", menciona un interno. Parafraseando a internos entrevistados, ellos ubican un cambio en la subjetividad de la población carcelaria, remarcando el carácter de improvisación y peligrosidad de los jóvenes que delinquen.

El personal penitenciario también resalta tal situación de cambio, en la cual lo que queda al descubierto es la "peligrosidad" en el accionar actual. Los jóvenes reclusos remarcan la carencia económica, el desvalimiento, la calle como socializadora a temprana edad, las drogas como paliativos a la angustia existencial personal, el sinsentido social y la construcción de un sentido en la "identidad antisocial". Le adjudican a ciertos estratos de poder de la sociedad también una conducta errática o transgresora, remarcando su particular situación de asimetría y vulnerabilidad social: "algunos sí pueden delinquir, si es que tienen dinero y/o poder", situación que desde el imaginario social es ratificada, avalando la falla de la legalidad.

Estas reflexiones nos conducen a establecer el foco de la investigación, el cual puede enunciarse a través de las siguientes preguntas: 1 . ¿Es posible resignificar, construir nuevos sentidos del trabajo en esta población particular? 2. ¿Cómo hacerlo?

\subsection{Las representaciones psíquicas}

Según Caza (2000), la idea de representación aparece en diversas teorías psicológicas, que coinciden en que lo que se representa es algo ausente. En la 
representación se pueden discernir dos elementos: el objeto ausente, por un lado, y aquello que lo representa, por el otro. Por ejemplo, Juan es el objeto ausente, y su foto es aquello que lo representa. Al primer elemento se lo llama "lo representado" y al segundo elemento "el representante". Lo representado es lo ausente, y el representante es lo presente.

En adelante, entonces, cuando se hable de representación se hará referencia a este último sentido, con lo cual se puede adelantar una definición preliminar del término: representación es todo aquello que sustituye o representa a un objeto que está ausente. Una palabra representa a una persona cuando se la nombra estando ausente, y lo mismo se puede decir de una imagen o un concepto. Esto nos lleva a la cuestión de los diferentes tipos de representación que se pueden encontrar. Teniendo en cuenta que el concepto de representación ha sido definido por diferentes marcos teóricos, en esta investigación se elige la perspectiva del modelo psicoanalítico.

La vertiente endopsíquica en relación con la representación, cuyo mentor es Freud (1915), caracteriza el concepto como el contenido ideaciones relativas a un objeto ausente. Distingue entre representaciones inconscientes y preconscientes-conscientes, y explica que en estas dos últimas opera la palabra, posibilitando la evocación de ese objeto o contenido ideaciones. Al respecto, Bleichmar (1990) señala que es el lenguaje el instrumento ordenador que posibilita acceder al conocimiento de estas representaciones psíquicas.

\section{El problema metodológico. El enfoque cualitativo}

La presente investigación se enmarca dentro del denominado enfoque cualitativo, el cual depende de la naturaleza de la pregunta de investigación y de la intencionalidad de la misma, dado que tal enfoque aborda las realidades subjetivas e intersubjetivas como objetos de conocimiento científico. Busca comprender, desde la interioridad de los actores sociales, las lógicas del pensamiento que guían las acciones sociales; estudia la dimensión interna y subjetiva de la realidad social como fuente de conocimiento; rescata la diversidad y particularidad haciendo especial énfasis en la valoración de lo subjetivo, lo vivencial y la interacción.

Como experiencia investigativa, el enfoque cualitativo hace énfasis en lo local, lo micro, lo regional; su objetivo es profundizar en la situación o problemática, y no necesariamente generalizar sus resultados. Los análisis cualitativos se centran en grupos pequeños, en casos o individuos que se seleccionan, 
El enfoque cualitativo en el estudio de representaciones sobre el trabajo en sujetos...

cuidando que no sean excepcionales, sino representativos. Trabaja con la palabra, argumentos, supuestos, gestos, representaciones, los rastros de cultura material.

Bottinelli (2003) señala que suscribir al uso de métodos cualitativos implica una búsqueda de comprensión desde el marco de referencia de aquel que actúa, sin que haya un control exhaustivo de todos los sesgos posibles. El investigador es considerado como un elemento que interviene y produce efectos en la investigación. Este tipo de estudios mantienen un abordaje sistémico, desde dentro; focalizan el proceso de un modo cíclico y recursivo, en un continuo en construcción - siempre parcial - del fenómeno, considerando el objeto como complejo, diverso y cambiante.

Lincoln y Guba (2000) describen las contribuciones del método cualitativo a los abordajes en ciencias sociales, destacando el retorno del sujeto y el cuestionamiento de la "verdad", sustituyéndola por la "verdad parcial", y la valorización de posturas más dialógicas con relación al rol del observador como parte de la investigación.

\section{Diseño de la investigación}

La investigación propuesta tiene carácter exploratorio y descriptivo, buscándose la caracterización de un fenómeno particular para plantear luego nuevas líneas de investigación. En su desarrollo, se intentan explorar las representaciones de sujetos privados de su libertad, en relación con un dispositivo específico de trabajo en el marco de un programa de reinserción social.

Por tratarse de una investigación de tipo cualitativa, se articulan las nociones teóricas que permiten esclarecer aspectos relativos a las representaciones, contextuadas con la noción de este dispositivo de trabajo particular en las organizaciones totales. Se intentará establecer las cualidades del cambio o la permanencia y estabilidad de las mismas.

Dado que el objeto de estudio de la presente investigación son las representaciones intrapsíquicas del trabajo - y en particular las construidas con relación al programa laboral de reinserción social que funciona en la Unidad 4 de la ciudad de Bahía Blanca - , los internos incluidos en estos programas de trabajo son definidos como la unidad de análisis. Para su incorporación en el programa del Servicio Penitenciario Bonaerense, se han tenido en cuenta criterios tales como el tipo de delitos por los cuales se encuentran penados o procesados, la conducta y el concepto institucional, una evaluación psico- 
lógica, el grado de reincidencia en el delito y la motivación para incluirse en el programa. A partir de entrevistas en profundidad en diferentes momentos cronológicos de la inclusión en los dispositivos laborales, se busca relevar eventuales modificaciones en las representaciones del trabajo de los sujetos incluidos en dicho dispositivo, explorando y describiendo la cualidad de esos cambios y su sentido.

El presente trabajo se encuentra incluido en los denominados estudios de caso. Ahora bien, para introducirnos en este tema se tendrá que definir en primer lugar qué se entiende por caso. Según Neiman y Quaranta (2006: 220), el caso "es definido como un sistema delimitado en tiempo y espacio de actores, relaciones e instituciones sociales donde se busca dar cuenta de la particularidad del mismo en el marco de su complejidad". En este trabajo, se definen como casos los relatos de un grupo de internos de la Unidad 4 de Bahía Blanca incluidos en un dispositivo laboral. A su vez, se establecieron ocho categorías de análisis con el objetivo de analizarlas en el desarrollo del trabajo de campo.

Tabla 1. Categorías de análisis

Sensación de pertenencia a un sector importante, necesario y valioso de la sociedad

Oportunidad para desarrollar las propias aptitudes

Espacio para el incremento y la adquisición del conocimiento

Interacción con otros y apoyo en relaciones interpersonales

Metas y asunción de roles y funciones

Plasmación de aspectos de la propia personalidad

Estructuración del tiempo

Retribución económica

Fuente: elaboración propia sobre la base de Kalimo (1988). 
El enfoque cualitativo en el estudio de representaciones sobre el trabajo en sujetos...

\subsection{Muestra}

Por las características que asume el enfoque de la investigación y para poder llegar a dar cuenta de los posibles cambios en las representaciones psíquicas ligadas al trabajo, este estudio prioriza aspectos tales como lo histórico y longitudinal por sobre la dimensión cantidad de sujetos.

En principio se ha establecido como criterio de selección de la muestra poblacional que el sujeto esté efectivamente incluido en el programa laboral, y se usó como criterio de exclusión que el delito por el cual cumple condena resulte contra la integridad sexual o la vida de las personas.

La muestra de la presente investigación está conformada por un total de cinco sujetos del programa laboral, a quienes se entrevistó utilizando el método de historia de vida. Se garantizó a los participantes su estado de anonimato, preservándose con extremo cuidado sus identidades. El período durante el cual se realizó la administración de las entrevistas abarcó desde el momento anterior a la inserción al programa laboral hasta seis meses después de su culminación. Sin embargo, existen variaciones con respecto al período considerado, debido a la posibilidad de obtención de un beneficio de tipo liberatorio por parte de los sujetos - que no es homogéneo en la muestra testeada - , por lo que el tiempo promedio estimado resultó ser de un año.

\subsection{El método: historia de vida}

La historia de vida corresponde a la metodología del "estudio de caso", se recoge durante un largo período de tiempo por aproximaciones sucesivas, debido a su amplitud. La historia de vida tiende a la exhaustividad y se suelen además emplear otros documentos o testimonios que corroboren o amplíen la información recogida. Es de carácter más interpretativo cuando conjugan datos de diferentes fuentes.

La utilización del método de la historia de vida se hace presente en las ciencias sociales desde su extensa utilización por la escuela de Chicago en 1920. Con el liderazgo de Robert E. Park, un grupo de investigadores conquistó gran popularidad para ese abordaje metodológico, que pasó a ser uno de los más utilizados en el Departamento de Sociología de esa universidad. Los estudios desarrollados sobre sujetos "desviantes" - denominándose así a los inmigrantes, jóvenes delincuentes, prostitutas - se articulaban para formar una especie de mosaico de la ciudad de Chicago en ese período. 
Como ya se ha mencionado, el presente trabajo busca comprender las representaciones psíquicas del trabajo de sujetos privados de libertad, incluidos en un dispositivo de trabajo en el marco de un programa de reinserción social. Por lo tanto, la historia de vida será el instrumento por excelencia, debido a que su nacimiento se encuentra ligado a los grupos "desviados", como se podría categorizar al objeto de estudio considerado en la presente investigación según esta perspectiva.

Tal contexto de investigación incluye la idea de acompañamiento, es decir, requiere que el objeto de estudio sea observado en diferentes momentos. Se hace entonces necesaria la utilización de diferentes técnicas que posibiliten ampliar la recolección de datos, complementando de esta manera el método de la historia de vida. Es indispensable establecer recurrencias y convergencias entre los datos, posibilitando de esta forma otorgarles consistencia y profundidad a los resultados que se obtengan en la investigación. Las historias de vida son construidas a partir de entrevistas cualitativas profundas, como lo establecen Mason (2002) y Bryman (2004). Su elaboración puede también incluir análisis documental (material escrito, diarios, otros documentos), y asimismo recurrir a la observación directa de la acción de los sujetos en sus condiciones concretas de vida.

Plummer (2001) ayuda a revalorizar las historias de vida como una estrategia innovadora que otorga a los individuos de carne y hueso una nueva voz. Sitúa claramente a las historias de vida en una posición en la cual pueden ser evaluadas científicamente, tras lo cual sus datos serían aprobados por los criterios cualitativos de amplitud, coherencia y parsimonia. Los datos aportados por la investigación biográfica aprobarían de la misma manera los criterios clásicos de validez, confiabilidad, replicabilidad y objetividad.

\subsection{La técnica: la entrevista}

En la presente investigación, se utiliza como técnica de recolección de datos la entrevista semidirigida en tanto permite - a través del análisis de los datos obtenidos - el acceso a las representaciones psíquicas del trabajo, así como a las percepciones de los empresarios sobre los trabajadores. Por lo tanto, la técnica se considera propicia para acceder al objeto de estudio, teniendo en cuenta además que en la actualidad no se cuenta con otras técnicas para acceder a las representaciones psicológicas del trabajo en procesos de investigación.

La entrevista puede ser definida como la conversación de dos o más personas en un lugar determinado para tratar un asunto (encuadre). Técnica- 
El enfoque cualitativo en el estudio de representaciones sobre el trabajo en sujetos...

mente, es un método de investigación científica que utiliza la comunicación verbal para recoger informaciones en relación con una determinada finalidad (Grawitz, 1984: 188; Aktouf, 1992: 91; Mayer y Ouellet, 1991: 308).

Lo que se comunica en una entrevista es más que aquello expresado; los silencios, el tono de voz, las recurrencias temáticas, la comunicación paraverbal, las emociones frente a diferentes tópicos son datos que al converger se convierten en información para el entrevistador.

\section{Discusión}

La metodología planteada facilita acceder a las representaciones psíquicas del trabajo, dado que tal objeto de estudio es inaccesible a través de metodologías cuantitativas. En palabras de Taylor y Bogdan (1986), el método cualitativo es un multimétodo interpretativo que permite estudiar a las personas en su ambiente natural. En tal sentido, las entrevistas se desarrollan en la unidad carcelaria, así como en las empresas en las cuales se desempeñan laboralmente los internos que forman parte del dispositivo específico analizado, accediendo a la obtención de datos a partir de cierto grado de inclusión del observador en el devenir institucional y del ámbito en el cual se desarrolla la actividad laboral.

$\mathrm{El}$ investigador es considerado como un elemento que interviene y produce efectos en la investigación. Es un aspecto a destacar que quien investiga en campo se desempeña laboralmente en la institución carcelaria, tal condición genera una modalidad particular al proceso, en tanto implica un conocimiento situado sobre la institución y una mayor proximidad con las unidades de análisis (sujetos privados de la libertad e incluidos en este dispositivo laboral).

Como experiencia investigativa, el enfoque cualitativo hace énfasis en lo local, lo micro. Su objetivo es profundizar el conocimiento de la situación, y no necesariamente generalizar sus resultados. Los análisis cualitativos se centran en grupos pequeños - en casos o individuos que se seleccionan como en la presente investigación - , y a su vez se realiza un estudio histórico y longitudinal para advertir los posibles cambios en las representaciones psíquicas de los internos. Se hace énfasis en la particularidad del objeto de estudio, se elige estudiar un caso. Es este énfasis en lo subjetivo, lo particular y la búsqueda de caracterizar las cualidades únicas de un objeto de estudio complejo (por su condición humana individual, social, histórica, simbólica, sujeto del lenguaje), lo que nos lleva a elegir la historia de vida, para la presente investigación, como método integral de abordaje del objeto de estudio, en el cual el foco no está en 
la representatividad de la muestra, sino en la lógica interna del discurso de los sujetos que integran estos programas.

\section{Críticas a la investigación cualitativa}

- Es particular sin permitir la generalización de sus resultados.

- Es lenta y dificultosa, exige estudios de tipo longitudinales para advertir en profundidad las cualidades del objeto de estudio y su evolución en el tiempo.

- Requiere un fuerte entrenamiento del investigador en la compresión de los fenómenos sociales.

- Involucra la subjetividad en el proceso de conocimiento pudiendo perderse la distancia óptima del objeto de estudio.

- Está más centrado en la relevancia del estudio que en el rigor metodológico.

Otro aspecto a ser considerado es la marcada subjetividad que implica el análisis de los datos obtenidos en las entrevistas. Es el investigador quien crea el instrumento y define las categorías de análisis, lo aplica en el campo y analiza los resultados con categorías relevadas en el discurso de los internos, para lograr así reconocer aquellas particulares aportadas por este grupo.

\section{Resultados}

Con respecto a la primera hipótesis - este dispositivo laboral de reinserción provoca un cambio en las representaciones del trabajo de los internos privados de su libertad - , considerando los resultados obtenidos en diferentes momentos del proceso de investigación, sobre la base de la experiencia de este grupo de internos, la principal conclusión reside en el efecto positivo del dispositivo laboral. En las diferentes categorías analizadas, se encuentran respuestas alentadoras, en pos del funcionamiento de este tipo de dispositivo laboral.

La segunda hipótesis es que el dispositivo laboral de reinserción se encuentra alineado, estableciéndose la serie de repetición, y que el trabajo de esta forma se convertiría en una actividad alienante. Los resultados muestran lo siguiente: 
El enfoque cualitativo en el estudio de representaciones sobre el trabajo en sujetos...

- La vivencia del tiempo de su condena se torna diferente en tanto hay un marco de organización temporal externo, en particular los horarios y las normativas de la empresa.

- Todos los internos reconocieron vivenciar efectos positivos como resultado de su inserción al dispositivo laboral; sin embargo, estos efectos no son suficientes para pensar en un resultado de resocialización. Cuatro de ellos no lograron finalizar su experiencia en el dispositivo de trabajo, produciéndose su desvinculación antes de lo esperado.

- Tres de ellos coinciden en que el trabajo en estas empresas les brindaba la sensación de pertenencia a un sector importante, necesario y valioso de la sociedad; los otros dos internos le atribuyen un efecto positivo, sin poner el acento en el reconocimiento social que pudiera percibirse de la firma. En uno de los casos se advierte desgano, y cierta falta de motivación en el trabajo.

- Toda la muestra indicó que su trabajo aportaba metas y asunción de roles y funciones, así también que facilitaba la estructuración del tiempo, evidenciándose un cambio importante en este sentido. Destacaron que sus actividades laborales les permitían la plasmación de aspectos de la propia personalidad. Uno de los internos hizo referencia a ciertas dificultades en la interacción con otros pares; los cuatro restantes señalaron percibir apoyo en las relaciones interpersonales.

- Coincidieron en que esta experiencia, habilitaba un espacio para el incremento y la adquisición del conocimiento, brindado la oportunidad para desarrollar las propias aptitudes.

- La dimensión retribución económica presentó conformidad por parte de cuatro de los entrevistados, en tanto que uno de ellos se mostró insatisfecho con el pago.

Considerando los objetivos de acción, se muestran a continuación los resultados obtenidos (tablas 2, 3 y 4 ). 
Tabla 2. Resultados del objetivo de acción n. ${ }^{\circ} 1$

Objetivo de acción n. ${ }^{\circ}$ 1. Establecer eventuales criterios de mejora a los programas actuales

- Se requiere del compromiso de las autoridades (director/a y subdirectores/a). Desarrollando políticas de tratamiento en los niveles de la gestión organizacional.

- Es necesario optimizar los procesos de selección en pos de disminuir los fallos en la inclusión y ajuste al ámbito laboral, así como su permanencia en estos espacios.

- También es necesario establecer un proceso de preparación anterior a la inserción laboral, basado en la capacitación y concientización, desde la Sección de Talleres de la unidad penal.

- Realizar una inducción formalizada por parte de las empresas que incluirán este tipo de trabajadores.

- Definir una política de comunicación externa, dirigida a las empresas locales en pos de obtener mayores resultados.

- Mejorar las condiciones laborales, estableciendo un contrato laboral (ART, seguro), y garantizar oficialmente la disminución de las cargas sociales que debe pagar el empresario. Que no presente el formato legal de beca de capacitación.

- Que el convenio este reconocido por el Ministerio de Nación.

Fuente: elaboración propia.

Tabla 3. Resultados del objetivo de acción $n .^{\circ} 2$

Objetivo de acción n. ${ }^{\circ}$ 2. Desarrollar políticas respecto del diseño de dispositivos de trabajo para los decisores en instituciones totales

- Es necesario optimizar la comunicación y articulación entre el interno, la empresa, y el juzgado a cargo. También gestionar ante el juzgado la solicitud del beneficio de salidas laborales. Y realizar un seguimiento y control del desempeño de los internos en las empresas y en el dispositivo institucional. 
El enfoque cualitativo en el estudio de representaciones sobre el trabajo en sujetos...

- Es destacable que este dispositivo facilita el aprendizaje de tareas, transferibles a otros ámbitos laborales. También se remarca que brinda sostén económico.

- Por las cualidades de los grupos laborales en los cuales se incluyen, potencialmente pueden obtener sostén emocional.

- Todas las empresas aportan metas de aprendizaje profesional, establecen objetivos de desempeño, en uno de los casos, el grupo de trabajo, posee la capacidad de regular los tiempos productivos, según los objetivos definidos.

- Permiten el desarrollo del potencial a través de aprendizajes en el trabajo.

- En este tipo de población es importante fomentar el desarrollo de diferentes aptitudes en lo profesional, lo social y lo humano.

- Señalan que insertarse en una estructura laboral les permiten de manera potencial múltiples aprendizajes.

- Los empresarios ligan tal convenio al concepto de Responsabilidad Social Empresaria (RSE).

Fuente: elaboración propia.

Tabla 4. Resultados del objetivo de acción n. ${ }^{\circ} 3$

Objetivo de acción n. ${ }^{\circ}$. Aportar conocimiento para la gestión de recursos humanos en las empresas incluidas en este tipo de programas

- Es necesario establecer la importancia de la preparación de los internos en términos de hábitos de trabajo y capacitación antes del ingreso al programa laboral.

- Muchos de los internos incorporados al programa no cuentan con hábitos y una cultura del trabajo, por lo que les cuesta sostener el trabajo. Suele haber reclamos de las empresas por faltas injustificadas.

- Un criterio que facilita su inclusión y ajuste a los requerimientos del programa laboral es el acompañamiento y contención de la familia.

- Para aquellos internos radicados en Bahía Blanca, suele ser auspicioso, pudiendo continuar en ese trabajo, o llegar a adquirir uno nuevo. 
- Todos los empresarios coincidieron en que este programa implica para sus empresas una forma de RSE; en algunos casos, se encuentra formalizada, como una política planificada y parte de la cultura organizacional, y en otros casos forma parte de los valores inherentes al compromiso social empresario de los propietarios.

- En principio, se advierte como factor motivacional que trabajan para salir del encierro y obtener un ingreso económico. En algunos casos, se observa la motivación de estar en otro entorno, dignificarse.

- Se generan aprendizajes, algunos con políticas formalizadas y otros desde la capacitación que se brinda en el puesto.

- Coincidieron en que el dispositivo brinda sostén y potencialmente genera condiciones para el crecimiento personal y profesional. Destacan que aporta roles definidos y metas a ser logradas, que organizan el trabajo.

- Este tipo de iniciativas aporta desde lo social, y tendería a disminuir la conflictividad laboral.

- Alguno de los empresarios destacan que este programa brinda una ventaja, el no pagar cargas sociales (aportes, ni obra social), debido a que es un sistema de capacitación laboral. Tal modalidad de contratación hace que disminuyan los costos productivos.

Fuente: elaboración propia.

\section{Conclusiones}

La presente investigación permite explorar y caracterizar los efectos del tratamiento penitenciario en la vida psíquica de un grupo etario (internos), aportando un conocimiento inédito. Brindará, además, un aporte a la administración desde un enfoque cualitativo y la aplicación poco usual, en este ámbito de conocimiento, del método historia de vida.

Se articulan lógicas diferentes y confluyentes tales como la del sistema penal, la subjetividad en el trabajo de internos incluidos en una institución de carácter total.

Los resultados obtenidos brindarán elementos a los directivos, o gestores, para la definición y desarrollo de políticas de tratamiento, como así también elementos valiosos para el desarrollo de prácticas ajustadas en recursos humanos a las diferentes empresas que trabajan con estos grupos. 
El enfoque cualitativo en el estudio de representaciones sobre el trabajo en sujetos...

Con respecto a futuras líneas de investigación, podrá indagarse sobre los posibles efectos de otros dispositivos de trabajo dentro de las cárceles y su potencial relación con distintas variables observadas desde la institución penal y judicial, tales como conducta institucional, capacitación en oficios, estudios escolares.

\section{Bibliografía}

Atour, O. (1992). Méthodologie des sciences sociales et approche qualitative des organisations. Sainte-Foy: Presses de l'Université du Québec.

Bryman, A. (2004). Social Research Methods (2. ${ }^{a}$ edition). Oxford: Oxford University Press, pp. 468-499.

Botticelli, M. (2003). Metodología de Investigación. Herramientas para un pensamiento científico complejo. Buenos Aires: Ediciones Autor.

Bleichmar, S. (1990). Lo inconsciente: fecundidad clinica de sus paradigmas en Lecturas de Freud Buenos Aires. Argentina: Editorial Lugar.

Caza, P. (2000). Representación y fantasía. Enfoques cognitivo y psicoanalítico. Buenos Aires: Biblioteca Redpsicología.

Constitución de la Nación Argentina. Sancionada por el Congreso General Constituyente. Reformas de 1860, 1866, 1898, 1957 y 1994 ordenado por ley 24430. Texto Oficial de 1 de Mayo 1853. Estudio comparativo con el articulado anterior. Tratados internacionales con jerarquía constitucional (1999). Buenos Aires: Editorial Astrea (9. ${ }^{a}$ edición actualizada y ampliada).

Foucault, M. (2008). Vigilar y castigar. Nacimiento de la prisión Buenos Aires. Buenos Aires: Siglo XXI (edición revisada).

Freud, S. (2007 [1915]). Obras Completas T XIV. Buenos Aires: Amorrortu Editores, pp. 153-213.

Freud, S. (2007 [1915]). Conferencias de Introducción al Psicoanálisis. En Obras Completas T XVI. Buenos Aires: Amorrortu Editores.

Grawitz, M. (1984). Métodos y técnicas de las ciencias sociales. México: Editia mexicana.

Ley Orgánica del servicio Penitenciario n. ${ }^{\circ}$ 9079. Decreto ley 9578/80. Junta Militar. La Plata 11 de Agosto de 1980. Texto actualizado con las modificaciones introducidas por la Ley 10158, 10316, 10358 y 11257. Disponible en www.gob.gba.gov.ar/legislacion/legislacion/1-9079.html

Ley de Ejecución Penal Bonaerense n. ${ }^{\circ}$ 12256. Decreto número 38/99. Sancionada por el Senado y Cámara de Diputados de la Provincia de Buenos 
Aires. Publicada en el boletín oficial. La Plata, 19 de enero de 1999. Texto actualizado con las modificaciones introducidas por la Ley 12543, 13177, 13254, 13710, 13892 y 14296. Disponible en www.gob.gba.gov.ar/legislacion/legislacion/1-12256.html

Marx, K., y Engels, F. (2011 [1848]) Manifiesto del partido comunista. Madrid: Alianza.

Mayer, R. y Ouellet, F. (1991). Métodologie de recherchepour les intervenantssociaux. Boucherville: Gaëtan Morin Éditeur.

Mason, J. (2002). Qualitative researching. London: Sage Publications, pp. 62-83.

Neiman, G. y Quaranta, G. (2006). Los estudios de caso en la investigación sociológica. En Vasilachis de Gialdino, I. (coord.). Estrategias de investigación cualitativa. Barcelona: Gedisa, pp. 213-238.

Taylor, S. y Bogdan R. (1986). Introducción a los métodos cualitativos de investigación. Buenos Aires: Paidós.

Plummer, K. (2001). Documents of Life 2: An invitation to a Critical Humanism. Londres: Sage Publications.

Sirvent, M. (1999). Problemática metodológica de la investigación educativa. Ateneos del IICE Revista del IICE, VIII (14).

Volnovich, J. (2000). Acerca de la exclusión social. Recuperado el 20/07/19 de http://www.topia.com.ar

Volnovich, J. (2003). Herramientas freudianas para la Argentina de hoy. Recuperado el 20/07/19 de http://www.topia.com.ar

(C) 2017 por los autores; licencia otorgada a la revista Escritos Contables y de Administración. Este artículo es de acceso abierto y distribuido bajo los términos y condiciones de una licencia Atribución-No Comercial 4.0 Internacional (CC BY-NC 4.0) de Creative Commons. Para ver una copia de esta licencia, visite https://creativecommons.org/licenses/by-nc/4.0/ 